\title{
Non-progressive familial idiopathic intracranial calcification: a family report
}

\author{
John S Callender
}

\begin{abstract}
The clinical features and long term outcome of familial idiopathic intracranial calcification in three members of one family are described. The illness presented as psychiatric disorder in all patients, and in one patient, epilepsy and intellectual deterioration were later manifestations. Skull radiographs and CT were performed sequentially, in one patient, over a 22 year period and, in another, CT was carried out eight years apart. In neither patient was there any evidence of progression of calcification.
\end{abstract}

(F Neurol Neurosurg Psychiatry 1995;59:432-434)

Keywords: familial intracranial calcification; clinical features; outcome

Intracranial calcification has been a finding in about $1 \%$ of patients examined by brain CT. ${ }^{12}$ One study of a psychiatric population found calcification of basal ganglia in $0.87 \%$ of a large series of patients of mixed diagnoses. ${ }^{3}$

Familial idiopathic intracranial calcification may present with neurological or psychiatric syndromes including functional psychoses and dementia. ${ }^{4}$ Inheritance may be autosomal dominant or $\mathrm{X}$ linked dominant. Casanova et $a l$, in another psychiatric series, found the incidence of basal ganglia mineralisation similar in schizophrenic and non-schizophrenic populations. ${ }^{5}$

A detailed histochemical study of a case of familial calcification was provided by Adachi $e t$ $a l .{ }^{6}$ The basal ganglia, the deep white matter, and the dentate nuclei of the cerebellum showed abundant mineral deposits consisting mainly of calcium and iron. Adjacent areas contained high levels of a non-mineralised acid mucopolysaccharide. The appearances suggested an origin of the abnormal material in the glial cells with extension into the pericellular areas.

A family is described in which intracranial calcification presented as psychiatric illness in a mother and her two sons. This family is of interest in that radiological examinations have been carried out on one affected member over a 22 year period and in another over eight years. The parents of the mother are reported to have died in old age of natural causes. The psychiatric hospital which serves their place of residence has no record of contact with them. There are no surviving general hospital case notes. The only surviving relative is the daughter of patient 3. She is currently aged 17 and has not yet presented to her local psychiatric service. All family members described in this report are, or were, of normal height, stature, and physical appearance.

\section{Case reports}

PATIENT 1

This woman was an only child. She first presented for psychiatric care at the age of 38 . There was a history of depression and abuse of drugs and alcohol dating back several years before presentation. She subsequently had many admissions to hospital as a result of depression and alcohol abuse. At other times she presented with features of promiscuity, aggression, and facetiousness. She made many attempts at suicide. She was diagnosed as having manic depressive psychosis. In her later years she became more stable and was maintained on phenothiazine medication. A mild degree of intellectual deterioration became apparent but formal psychometric testing was not performed. She died at the age of 56 of causes unrelated to her psychiatric disorder. Calcification of the basal ganglia was detected by skull radiography performed when the patient was 47 years old (figure). Serum calcium and phosphorus were normal as were all other routine investigations.

\section{PATIENT 2}

This 37 year old man first presented in his mid-teens with a history of social withdrawal, decline in academic performance, and bizarre behaviour. In subsequent months, he developed paranoid delusions and auditory hallucinations. A diagnosis of schizophrenia was made and he was treated with neuroleptic medication. His condition has proved refractory to treatment and he is now an inpatient in a long term psychiatric ward. He continues to be troubled with delusions and hallucinations. $\mathrm{He}$ exhibits motor symptoms such as choreiform movements, stereotyped spinning, and echopraxia. His thinking is impoverished and very disordered. $\mathrm{He}$ is apathetic and socially isolated. 


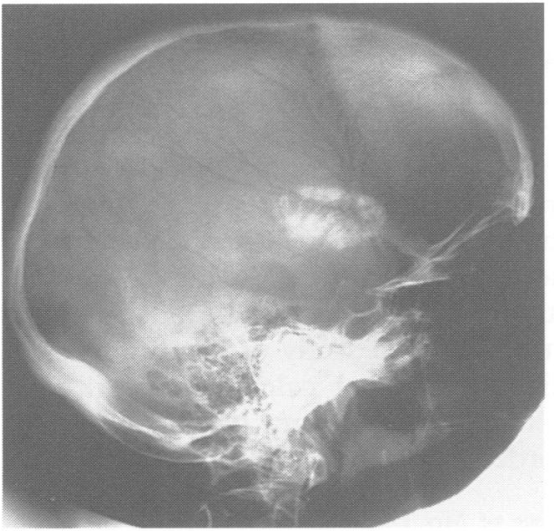

(A)

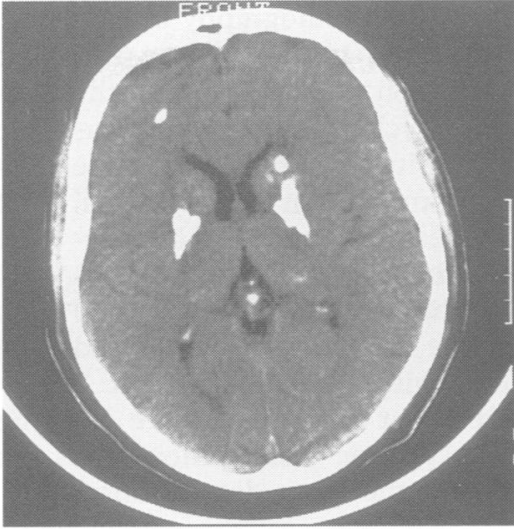

(B)

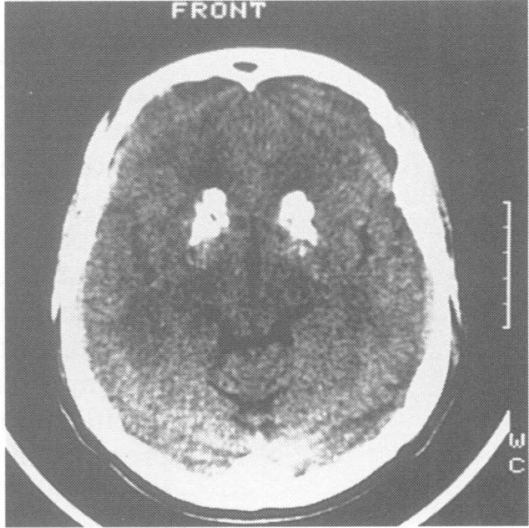

(C)

(A) Skull radiograph of patient 1, age 47. (B) CT of patient 2, age 37. (C) CT of patient 3, age 43.

A skull radiograph, performed at the age of 15 after the onset of his bizarre behaviour, disclosed no abnormality. Eight years later bilateral basal ganglia calcification was revealed on a skull radiograph. After this, CT was performed and calcification in the basal ganglia was confirmed, along with small spots of calcification in the anterior cerebral substance. Repeat scans performed eight and 14 years later showed no change (figure). Cerebral blood flow studies using ${ }^{99 \mathrm{~m}} \mathrm{Tc}$ pertechnetate performed at the time of the most recent scan were reported as normal. An EEG at age 15 was normal; eight years later he began to experience grand mal fits. At this time, he was taking haloperidol and procyclidine. A subsequent EEG showed excess slow wave activity. Routine haematological and biochemical investigations have been normal as have estimations of serum caeruloplasmin, thyroid function, syphilis serology, and the CSF Lange curve. His serum calcium and phosphate are normal. A modified Ellsworth Howard test was performed which showed a normal cyclic AMP and phosphaturic response to parathyroid hormone. Radiographs of his hands and long bones showed no abnormality.

Sequential intellectual testing showed a progressive decline in verbal and performance IQ between the ages of 14 and 23, taking him from a dull-normal level of functioning to one characteristic of moderate mental deficiency. In the subsequent nine year period no further deterioration occurred.

PATIENT 3

This patient was first referred for psychiatric care at the age of 26, complaining of depression and irritability. He was seen on one occasion and discharged from care. $\mathrm{He}$ was rereferred at age 44 . In the years before this he had been noted to exhibit odd behaviour in the course of visits to his brother in hospital. His wife had left him because of repeated, remorseless violence. Two months after referral he was admitted to hospital after attempting suicide.

On admission to hospital he exhibited blunting and incongruity of mood. He described various bizarre delusional ideas. He was diagnosed as schizophrenic. His illness responded poorly to medication. He eventually absconded from hospital and committed suicide by vehicle exhaust gas poisoning.

Computed tomography was performed at age 35 and again eight years later (figure). Dense calcification was present bilaterally in the basal ganglia. There were also specks of calcification in the frontal and parietal lobes. No progression was noted between the two examinations. Routine haematological and biochemical investigations including serum calcium and thyroid function tests were normal.

\section{Discussion}

This family is of interest in view of the opportunity presented for repeated assessment of pathological changes by CT over a period of 14 years in one affected family member and eight years in his brother. There has been no clinical or biochemical evidence of idiopathic hypoparathyroidism or other relevant illness in any affected family member and pseudohypoparathyroidism was excluded in patient 2 .

The clinical picture was of a functional psychiatric disorder in patients 1 and 3, although the pattern of symptoms presented was strikingly dissimilar. Patient 2 has shown a mixed picture of severe schizophrenic illness, epilepsy, and intellectual deterioration. In all cases, the response to antipsychotic medication was poor. The onset of radiologically apparent calcification occurred in patient 2 between the ages of 15 and 23, after which there was no further progression. Between the ages of 14 and 23 there was a substantial decline in intellectual function which then remained static. In patient 3 there was no progression of calcification on CT between the ages of 35 and 43.

The relation between calcification and psychopathology in this family is suggestive but by no means conclusive. In patient 2 psychiatric symptoms were present at a time when his skull radiograph was normal. In the series reported by Harrington et al, however, calcification was visualised on CT in 26 patients but 
this was apparent on skull radiography in only seven. ${ }^{2}$ It may, therefore, be the case in this patient that calcification was present at the time of onset of his psychiatric illness but was not of sufficient density to show on routine radiography. The combination of functional psychosis, epileptic seizures, and early intellectual deterioration, which seems to have developed in parallel with calcification, is unusual and suggests that the calcification has played an important aetiological part. In patient 3 calcification was present for years before his presentation in a psychotic state although it is difficult to pinpoint the exact date of onset of this.

The diversity of clinical manifestations of familial intracranial calcification in case reports is striking and this diversity has led some to question the validity of familial intracranial calcification as an entity and a cause of psychosis. ${ }^{3-5}$

The clinical manifestations of intracranial calcification may be diverse and dependent on which parts of the nervous system bear the brunt of the pathological process, but also in the modulation of these effects by the personality of the patient, his intelligence, past experience, and his age and level of maturation at the time of onset of the symptoms. Damage to the basal ganglia may lead to complex behavioural abnormalities, and it has been suggested that this is due to loss of a "sensory gating" function in the basal ganglia. ${ }^{7}$
The precise localisation of intracranial calcification varies in different reports. ${ }^{34}$ In most of these, calcification has been determined by skull radiography and as indicated this may give an inadequate picture of the extent of calcification. At present it is difficult to draw any clear conclusions on the relation between location of lesions and clinical manifestations. As information accumulates using modern $\mathrm{CT}$, it may be that detailed study of conditions such as familial intracranial calcification will cast light on the neuroanatomy of major psychiatric disorders such as schizophrenia.

The assistance of Mrs Diane Sandison in typing the manuscript, and of the Department of Medical Illustration, Medical School, Foresterhill, Aberdeen, in preparing the figures, is gratefully acknowledged.

1 Brannan TS, Burger AA, Chaudhary MY. Bilateral basal ganglia calcification visualised on CT scan. $\mathcal{f}$ Neuro Neurosurg Psychiatry 1980;43:403-6.

2 Harrington MG, Macpherson P, McIntosh WB, Allan BF Bone I. The significance of the incidental finding of basal ganglia calcification on computed tomography. $f$ Neurol Neurosurg Psychiatry 1981;44:1168-70.

3 Philpot MP, Lewis SW. The psychopathology of basal ganglia calcification. Behavioural Neurology 1989;2:227-34.

4 Flint J, Goldstein LH. Familial calcification of the basa ganglia: a case report and review of the literature. Psychol Med 1992;22:581-95.

5 Casanova MF, Prasad CM, Waldman I, et al. No difference in basal ganglia mineralization between schizophrenic and non-schizophrenic patients: a quantitative computerised tomographic study. Biological Psychiatry 1990;27: 138-42.

6 Adachi M, Wellmann KF, Volk BW. Histochemical studies on the pathogenesis of idiopathic, non-arteriosclerotic cerebral calcification. $\mathcal{F}$ Neuropathol Exp Neurol 1968;27: cerebral

7 Schneider JS. Basal ganglia role in behavior: Importance of sensory gating and its relevance to psychiatry. Biological Psychiatry 1984;19:1693-710. 